\title{
Algebras of Discrete Symmetries and Supersymmetries for the Schrödinger-Pauli Equation
}

Anatoly NIKITIN

Institute of Mathematics of the National Academy of Sciences of Ukraine,

3 Tereshchenkivs'ka Street, Kiev-4, Ukraine

It is shown that the Schrödinger-Pauli (SP) equation is invariant with respect to algebra $g l(4, C)$ provided the vector-potential of an external field has definite parities. This invariance algebra is used to reduce the SP equation to uncoupled subsystems and to search for extended and generalized supersymmetries.

PACS numbers: 11.30Ly - other internal and higher symmetries;

11.30.Pb - supersymmetry;

03.65.Fd - algebraic methods;

33.10.Cs - calculational methods (including new

theoretical techniques and applications of group theory). 


\section{Introduction}

Many of prosperous approaches to investigation of partial differential equations include search for their symmetries. In addition to many other important applications, symmetries often make it possible to reduce a given problem to a more simple one or even to construct its exact solution [1].

Such a reduction can be carried out using discrete symmetries like parity, charge conjugation, or rotations on angle $\pi$. The algebraic structures generated by these symmetries for the Dirac equation where studied in papers $[2,3]$.

Discrete symmetries present effective tools in searching for models which admit supersymmetry (SUSY) and generalized SUSY [4]. The idea to use the parity operator to construct supercharges was proposed long time ago [5], recently this approach was generalized and applied to search for extended SUSY in quantum mechanical problems, refer to ref. [2,6,7]. A specific feature of such generalization is using combined parities and rotations as structure elements of supercharges.

In the present paper we investigate discrete symmetries of the basic equations of the nonrelativistic quantum mechanics, i.e., the Schrödinger and Schrödinger-Pauli (SP) equations. The algebraic structures of these symmetries are analyzed, the complete list of the related reductions of the SP equation is presented. A new class of problems for the SP equation is found which generate extended SUSY.

\section{Discrete symmetries}

Let us investigate symmetry properties of the SP equation

$$
L \psi=0, \quad L=i \frac{\partial}{\partial x_{0}}-\frac{1}{2 m}\left(\pi^{2}-e g \vec{\sigma} \cdot \vec{H}\right)-e A_{0}
$$

(where $\pi^{2}=\pi_{1}^{2}+\pi_{2}^{2}+\pi_{3}^{2}, \quad \pi_{a}=-i \frac{\partial}{\partial x_{a}}-e A_{a}, \quad a=1,2,3, \quad e \vec{H}=-i \vec{\pi} \times \vec{\pi}, \quad \vec{\sigma}=$ $\left(\sigma_{1}, \sigma_{2}, \sigma_{3}\right), \sigma_{a}$ are Pauli matrices) w.r.t. the following discrete transformations of independent variables

$$
x_{\mu} \rightarrow r_{\nu} x_{\mu}=x_{\mu}\left(1-2 \delta_{\mu \nu}\right), \quad \mu, \nu=0,1,2,3 .
$$

We will use the notation $x \rightarrow r_{\nu} x$ for transformations of four-vector $x=\left(x_{0}, x_{1}, x_{2}, x_{3}\right)$ provided its components are transformed in accordance with (2).

Equation (1) is invariant w.r.t. a transformation $x \rightarrow r_{0} x$ or $x \rightarrow r_{a} x$ for a fixed $a=1,2,3$ provided the parity of the vector-potential $A=\left(A_{0}, A_{2}, A_{2}, A_{3}\right)$ is given by one of the following relations

$$
A\left(r_{0} x\right)=-r_{0} A(x)
$$

or

$$
A\left(r_{a} x\right)=r_{a} A(x)
$$

respectively. The action of matrices $r_{0}$ and $r_{a}$ on the four-vector $A$ is defined in the same way as in the case of the four-vector $x$. 
The corresponding transformation for the wave function $\psi(x)$ has the form

$$
\psi(x) \rightarrow \Gamma_{\mu} \psi(x)
$$

where $\Gamma_{0}=i \sigma_{2} c \theta_{0}, \quad \Gamma_{a}=\sigma_{a} \theta_{a}, \quad a=1,2,3$ (no sum over a), $\theta_{\mu}$ and $c$ are operators of reflection and complex conjugation:

$$
\theta_{\mu} \psi(x)=\psi\left(r_{\mu} x\right), \quad c \psi(x)=\psi^{*}(x) .
$$

Indeed, operators $\Gamma_{\mu}$ satisfy the condition $\left[L, \Gamma_{\mu}\right]=0$ and so transform solutions of (1) into other solutions of this equation.

Consider the 16-dimensional finite group $G$ generated by reflections (2) and their products. The related symmetries of equation (1) are expressed via products of operators $\Gamma_{\mu}$ (4) and form a projective unitary and antiunitary [8] representation of group $G$ with the following basis elements

$$
S_{5 \mu}=\frac{1}{2} \Gamma_{\mu}, S_{54}=\frac{1}{2} \Gamma_{4}=\frac{1}{2} \Gamma_{0} \Gamma_{1} \Gamma_{2} \Gamma_{3}, S_{4 \mu}=\frac{1}{2} \Gamma_{4} \Gamma_{\mu}, S_{\mu \nu}=\frac{1}{2} \Gamma_{\mu} \Gamma_{\nu}, I
$$

where $I$ is the unit operator.

Equation (1) admits all symmetries (6) provided the related vector-potential satisfies all relations (3) simultaneously.

Taking into account that operators (4) satisfy the Clifford algebra $\Gamma_{k} \Gamma_{l}+\Gamma_{l} \Gamma_{k}=2 g_{k l}$ where $g_{00}=g_{11}=g_{22}=g_{33}=-g_{44}=1, \quad g_{k l}=0, \quad k \neq l$, we conclude that symmetries (6) satisfy the following commutation relations

$$
\left[S_{k l}, S_{m n}\right]=g_{k n} S_{l m}+g_{l m} S_{k n}-g_{k m} S_{l n}-g_{l n} S_{k m}
$$

with $k, l, m, n,=0,1, \ldots, 5$ and $\quad g_{55}=-1$.

In accordance with $(7)$ operators $S_{k l}$ realize a representation of the Lie algebra $s o(3,3) \sim \operatorname{sl}(4, R)$. This algebra can be extended by adding products of symmetries (6) and the imaginary unit $i, i^{2}=-1$. Taking into account that $i=\Gamma_{1} \Gamma_{2} \Gamma_{3} P$ and $[P, L]=0$ where $P=\theta_{1} \theta_{2} \theta_{3}$, we obtain the additional set of symmetries

$$
\hat{S}_{\mu \nu}=P S_{\mu \nu}, \quad P
$$

whose commutation relations have the form

$$
\begin{aligned}
{\left[\hat{S}_{k l}, \hat{S}_{m n}\right] } & =g_{k n} S_{l m}+g_{l m} S_{k n}-g_{k m} S_{l n}-g_{l n} S_{k m} \\
{\left[\hat{S}_{k l}, S_{m n}\right] } & =g_{k n} \hat{S}_{l m}+g_{l m} \hat{S}_{k n}-g_{k m} \hat{S}_{l n}-g_{l n} \hat{S}_{k m} \\
{\left[S_{k l}, I\right] } & =\left[\hat{S}_{k l}, I\right]=\left[S_{k l}, P\right]=\left[\hat{S}_{k l}, P\right]=0 .
\end{aligned}
$$

Relations (7), (9) define a 32-dimensional Lie algebra which is equivalent to $g l(4, C)$.

Thus, if parities of the vector-potential satisfy all relations (3a) and (3b), the corresponding SP equation admits the symmetry algebra $g l(4, C)$. An example of the external field with such properties is the superposition of retarded and advanced plane waves with 
$A_{1}=A_{2}=0, \quad A_{3}=\phi\left(x_{0}-x_{3}\right)-\phi\left(x_{0}+x_{3}\right), \quad A_{0}=\phi\left(x_{0}-x_{3}\right)+\phi\left(x_{0}+x_{3}\right)$, where $\phi$ is an arbitrary even function, $\phi(-y)=\phi(y)$.

It follows from (3) that

$$
\begin{gathered}
A\left(r_{0} r_{a} x\right)=-r_{0} r_{a} A(x), \\
A\left(r_{a} r_{b} x\right)=r_{a} r_{b} A(x), \\
A(r x)=-r A(x), \\
A\left(r r_{0} x\right)=r r_{0} A(x), \\
A\left(r r_{a} x\right)=-r r_{a} A(x)
\end{gathered}
$$

where $r=r_{0} r_{1} r_{2} r_{3}$. Supposing that the vector-potential satisfies only a part of relations (3), (10), we reduce the related symmetry of the SP equation. The corresponding symmetry algebra is a subalgebra of $g l(4, C)$.

In Sections 4 and 5 we use the symmetries described in the above in order to reduce the SP equation.

\section{Stationary SP equation}

Consider now the stationary SP equation

$$
\frac{1}{2}\left(\pi^{2}-e g \vec{\sigma} \cdot \vec{H}+e A_{0}\right) \psi=E \psi
$$

where $E$ is an eigenvalue of the Hamiltonian, and external field is supposed to be time independent: $A=A(\vec{x}), \quad \vec{x}=\left(x_{1}, x_{2}, x_{3}\right)$.

The number of independent variables for equation (11) is reduced to 3 . The corresponding finite group of reflections of spatial variables is 8-dimensional and includes the following representativities

$$
\vec{x} \rightarrow r_{a} \vec{x}, \quad \vec{x} \rightarrow r_{a b} \vec{x}=r_{a} r_{b} \vec{x}, \quad \vec{x} \rightarrow \hat{r} \vec{x}=-\vec{x}, \quad \vec{x} \rightarrow I \vec{x}=\vec{x},
$$

where $a=1,2,3, r_{a}$ generates the reflection of the component of $\vec{x}$ with number $a$, etc.

In order to equation (11) be invariant w.r.t. one of transformations (12), $A_{0}(\vec{x})$ has to be invariant under this transformation, and $\vec{A}(\vec{x})$ has to have a definite parity. All these parities and the related symmetries of equation (11) are given by the following equations

$$
\begin{gathered}
\vec{A}\left(r_{a} \vec{x}\right)=r_{a} \vec{A}(\vec{x}), \quad P_{a}=\Gamma_{a}, \\
\vec{A}\left(r_{a b} \vec{x}\right)=r_{a b} \vec{A}(\vec{x}), \quad P_{a b}=\Gamma_{a} \Gamma_{b}, \\
\vec{A}(-\vec{x})=-\vec{A}(\vec{x}), \quad P=\theta_{1} \theta_{2} \theta_{3}, \\
\vec{A}\left(r_{a} \vec{x}\right)=-r_{a} \vec{A}(\vec{x}), \quad \hat{P}_{a}=C \Gamma_{a}, \\
\vec{A}\left(r_{a b} \vec{x}\right)=-r_{a b} \vec{A}(\vec{x}), \quad \hat{P}_{a b}=C \Gamma_{a} \Gamma_{b}, \\
\vec{A}(-\vec{x})=\vec{A}(\vec{x}), \quad \hat{P}=C \theta_{1} \theta_{2} \theta_{3} .
\end{gathered}
$$


Here $C=i \sigma_{2} c, \quad \Gamma_{a}, \theta_{a}$ and $c$ are defined in (4), (5).

If $\vec{A}(\vec{x})$ has definite parities w.r.t. two of transformations (12), it also has the fixed parity w.r.t. the product of these transformations. The related equation (11) is invariant under the three-dimensional Lie algebra of discrete transformations. If, for example, two of relations (13a) are satisfied (we will refer to this case as "(13a)+(13a)"), i.e.,

$$
\vec{A}\left(r_{a} \vec{x}\right)=r_{a} \vec{A}(\vec{x}), \quad \vec{A}\left(r_{b} \vec{x}\right)=r_{b} \vec{A}(\vec{x}), \quad a \neq b,
$$

then equation (11) admits the symmetry algebra so(3) generated by $\frac{i}{2} \Gamma_{a}, \quad \frac{i}{2} \Gamma_{b}$ and $-\frac{1}{2} \Gamma_{a} \Gamma_{b}$. In analogous way we find that the symmetry algebra so(3) corresponds also to the case of parities $(13 \mathrm{~b})+(13 \mathrm{~b})$ which are defined in analogy with (15). For the parities $(13 a)+(14 a),(13 a)+(14 c),(13 c)+(14 a),(14 a)+(14 a)$ and $(13 b)+(14 b)$ the related symmetry algebras are isomorphic to $s o(1,2)$, and for the case $(13 \mathrm{~b})+(13 \mathrm{~b})$ we have the Abelian algebra of discrete symmetries.

The most extended symmetry algebras correspond to the cases when $\vec{A}(\vec{x})$ admits three independent conditions from the set (13), (14). If conditions (13a) are satisfied for all $a=1,2,3$ (the case " $(13 \mathrm{a})+(13 \mathrm{a})+13 \mathrm{a}) "$, i.e.,

$$
A\left(r_{1} x\right)=r_{1} A(x), \quad A\left(r_{2} x\right)=r_{2} A(x), \quad A\left(r_{3} x\right)=r_{3} A(x)
$$

then the related equation (11) admits the symmetry algebra $s o(4) \oplus u(1)$ whose basis elements are

$$
S_{4 a}=\frac{i}{2} \Gamma_{a}, \quad S_{a b}=\frac{1}{2} \Gamma_{a} \Gamma_{b}, \quad I=i P .
$$

For the cases when parities of $\vec{A}(\vec{x})$ are of the types $(13 a)+(13 a)+(14 a)$, $(13 a)+(14 b)+(14 b)$ or $(14 b)+(14 b)+(14 b)$, the corresponding symmetry algebra for equation (11) reduces to $s o(1,3) \oplus d(1)$, so $(2,2) \oplus u(1)$ or $s o(1,3) \oplus u(1)$ respectively. Let us present the related sets of symmetries explicitly:

$$
\begin{gathered}
\vec{A}\left(r_{a} \vec{x}\right)=r_{a} \vec{A}(\vec{x}), \quad \vec{A}\left(r_{b} \vec{x}\right)=r_{b} \vec{A}(\vec{x}), \quad \vec{A}\left(r_{c} \vec{x}\right)=-r_{c} \vec{A}(\vec{x}), \\
S_{01}=\frac{i}{2} C \Gamma_{c}, \quad S_{02}=\frac{1}{2} C \Gamma_{b} \Gamma_{c}, \quad S_{03}=\frac{1}{2} C \Gamma_{a} \Gamma_{c}, \\
S_{12}=-\frac{i}{2} \Gamma_{b}, \quad S_{13} \frac{i}{2} \Gamma_{a}, \quad S_{23}=\frac{1}{2} \Gamma_{b} \Gamma_{a}, \quad I=C P ; \\
\vec{A}\left(r_{a} \vec{x}\right)=r_{a} \vec{A}(\vec{x}), \quad \vec{A}\left(r_{b} \vec{x}\right)=-r_{b} \vec{A}(\vec{x}), \quad \vec{A}\left(r_{c} \vec{x}\right)=-r_{c} \vec{A}(\vec{x}), \\
S_{12}=\frac{i}{2} \Gamma_{a}, \quad S_{34}=\frac{1}{2} \Gamma_{b} \Gamma_{c}, \quad S_{13}=\frac{1}{2} C \Gamma_{b}, \\
S_{14}=\frac{1}{2} C \Gamma_{c}, \quad S_{23}=\frac{i}{2} C \Gamma_{a} \Gamma_{b}, \quad S_{24}=-\frac{i}{2} C \Gamma_{a} \Gamma_{c}, \quad I=i C P ; \\
\vec{A}\left(r_{a} \vec{x}\right)=-r_{a} \vec{A}(\vec{x}), \quad a=1,2,3 \\
S_{0 a}=\frac{1}{2} C \Gamma_{a}, \quad S_{a b}=\frac{1}{2} \Gamma_{a} \Gamma_{b}, \quad I=i C P .
\end{gathered}
$$

We see that if the vector-potential of an external electromagnetic field has combined parities w.r.t. transformations (12), the corresponding stationary SP equation is characterized by internal symmetry algebras which cause degenerations of energy values. It is possible to show that the most extended (four times) degeneration corresponds to the 
cases described by relations (16), (18), (19) and (20). The examples of external fields satisfying these relations are given by the followimg formulae:

$$
\begin{gathered}
A_{1}=\frac{a^{2} m}{4 \pi} \frac{x_{1}\left(x^{2}-x_{2}^{2}\right)}{x^{7}}, \quad A_{2}=-\frac{a^{2} m}{4 \pi} \frac{x_{2}\left(x^{2}-x_{1}^{2}\right)}{x^{7}}, \quad A_{3}=0 ; \\
A_{1}=A_{2}=0, \quad A_{3}=-\frac{I}{4 \pi} \ln \left(x_{1}^{2}+x_{2}^{2}\right) ; \\
A_{1}=A_{2}=0, \quad A_{3}=-\frac{I}{4 \pi} \ln \frac{\left(x_{1}-b\right)^{2}+x_{2}^{2}}{\left(x_{1}+b\right)^{2}+x_{2}^{2}} ;
\end{gathered}
$$

and

$$
A_{1}=A_{2}=0, \quad A_{3}=-\frac{I}{4 \pi} \ln \frac{\left[\left(x_{1}+b\right)^{2}+\left(x_{2}+b\right)^{2}\right]\left[\left(x_{1}-b\right)^{2}+\left(x_{2}-b\right)^{2}\right]}{\left[\left(x_{1}+b\right)^{2}+\left(x_{2}-b\right)^{2}\right]\left[\left(x_{1}-b\right)^{2}+\left(x_{2}+b\right)^{2}\right]}
$$

Vector-potentials (21), (22), (23) and (24) correspond to the fields of magnetic octopole [7], infinite stright condactor with a constant current $I$, directed along the third coordinate axis, two stright conductors with opposite constant currents directed along the third co-ordinate axis and shifted to the distance $b$ w.r.t. $x_{1} x_{3}$ plane, and four stright condactors (two neihbouring ones have opposit currents) directed along the third coordinate axis and shifted to the distance $b$ w.r.t. the coordinate axis $x_{1}$ and $x_{2}$, see figers $a, b, c$ and $d$ respectively.

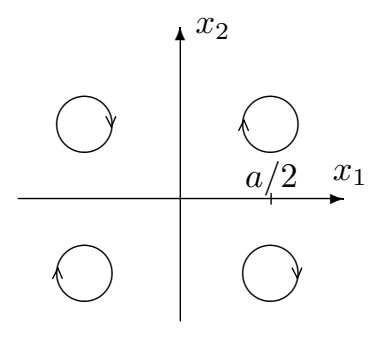

$a$

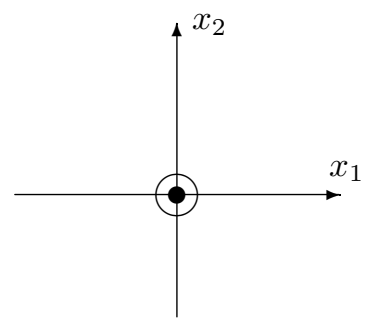

$b$

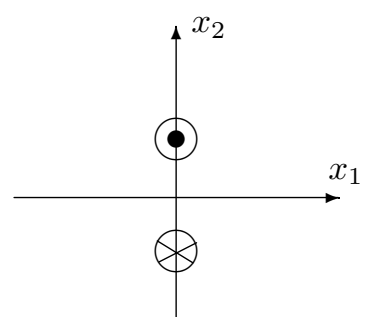

C

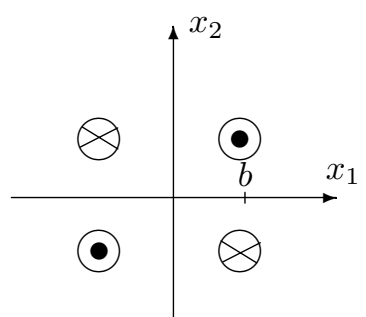

$d$

\section{Reduction of the SP equation}

The symmetries found above can be applied to reduce the SP equation to uncoupled subsystems. Here we consider an example of such reductions.

Let the vector-potential $A(x)$ satisfies relations (3b) for $a=1$. In this case equation (1) admits the symmetry $2 S_{51}=\sigma_{1} r_{1}$.

Using the operator

$$
U=\frac{1}{\sqrt{2}}\left(1+\sigma_{3} S_{51}\right), \quad U^{-1}=U^{\dagger}=\frac{1}{\sqrt{2}}\left(1-\sigma_{3} S_{51}\right)
$$

we reduce $2 S_{51}$ to the diagonal matrix form

$$
U 2 S_{51} U^{\dagger}=\sigma_{3}
$$


The corresponding transformed equation (1) looks as

$$
L^{\prime} \psi^{\prime}=0, \quad L^{\prime}=U L U^{\dagger}, \quad \psi^{\prime}=L \psi
$$

It follows from the above that $\left[L^{\prime}, \sigma_{3}\right]=0$ and so in accordance with Schur's lemma $L^{\prime}$ is diagonal too. Indeed, by direct calculation we obtain

$$
L^{\prime}=i \frac{\partial}{\partial x_{0}}-\frac{\pi^{2}}{2 m}-e g \sigma_{3}\left(H_{1} \theta_{1}+H_{3}\right)+i \theta_{1} H_{2}-e A_{0},
$$

i.e., equation (27) decouples to two independent equations for $\psi_{+}=\frac{1}{2}\left(1+\sigma_{3}\right) \psi^{\prime}$ and $\psi_{-}=\frac{1}{2}\left(1-\sigma_{3}\right) \psi^{\prime}$

Let the vector-potential satisfies one more parity condition, say (10a) for $a=2$. The corresponding symmetry $2 S_{42}=-i c \theta_{0} \theta_{2}$ commutes with $S_{51}$ and so we can diagonalize $S_{51}$ and $S_{42}$ simultaneously. Using for this purpose the operator

$$
W=U_{2} U_{1}, \quad U_{2}=\frac{1}{\sqrt{2}}\left(1+i \theta_{0} \theta_{2}\right)
$$

we obtain

$$
\begin{gathered}
W 2 S_{51} W^{\dagger}=\sigma_{3}, \quad W 2 S_{42} W^{\dagger}=c, \\
L^{\prime \prime}=W L W^{\dagger}= \\
i \frac{\partial}{\partial t}+\Delta-\theta_{0} \theta_{2}(\vec{\nabla} \cdot \vec{A}+\vec{A} \cdot \vec{\nabla})-e^{2} \vec{A}^{2}+e A_{0} \\
+e g \sigma_{3}\left(H_{1} \theta_{1}+H_{3}\right)+\theta_{0} \theta_{1} \theta_{2} H_{2}
\end{gathered}
$$

where $\Delta$ and $\vec{\nabla}$ are the Laplace and gradient operators.

In accordance with (30) the transformed equation (1) $L^{\prime \prime} \psi^{\prime \prime}=0, \quad \psi^{\prime \prime}=W \psi$, reduces to four uncoupled equations for $\operatorname{Re} \frac{1}{2}\left(1 \pm \sigma_{3}\right) \psi$ and $\operatorname{Im} \frac{1}{2}\left(1 \pm \sigma_{3}\right) \psi$.

If, in addition, the vector-potential satisfies relations (10b) for $a=2, b=3$ then equation (1) admits three commuting symmetries $S_{51}, S_{42}$ and $P$. Using again transformation (30), we reduce (1) to eight uncoupled equations for $\operatorname{Re} \psi_{\epsilon}^{\nu}$ and $\operatorname{Im} \psi_{\epsilon}^{\nu}$, where $\psi_{\epsilon}^{\nu}=\frac{1}{4}\left(1+\nu \sigma_{3}\right)(1+\epsilon P) \psi^{\prime \prime}, \quad \nu, \epsilon= \pm 1$.

\section{Complete list of reductions and subalgebras of algebra $g l(4, C)$}

Thus, to reduce equation (1) to uncoupled subsystems it is sufficient to diagonalize the related set of commuting symmetries (6), (8) which are admitted by this equation.

It is necessary to note that only a part of discrete symmetries discussed in Section 2 can be used to reduce equation (1). Some of them, say $S_{50}$, cannot be transformed to a diagonal matrix form. In addition, without loss of generality it is possible to reduce the number of symmetries which generate reductions, using the equivalence relations

$$
\hat{S}_{4 k}=U S_{0 k} U^{\dagger}, \quad S_{4 k}=U \hat{S}_{0 k} U^{\dagger}
$$

where $U=\frac{1}{\sqrt{2}}\left(1+\Gamma_{4} \Gamma_{0} P\right)$ is the operator commuting with $L$ of (1) for any vectorpotential $A(x)$. 
To describe effectively all reductions generated by discrete symmetries (6), (8) we use the isomorphism of algebra (7), (9) to the matrix algebra $g l(4, C)$. This isomorphism can be established by the following relations

$$
2 S_{k l} \leftrightarrow \tilde{\gamma}_{k} \tilde{\gamma}_{l}, 2 S_{5 k} \leftrightarrow \tilde{\gamma}_{k}, 2 \hat{S}_{k l} \leftrightarrow \tilde{\gamma}_{k} \tilde{\gamma}_{l} \tilde{P}, 2 \hat{S}_{5 k} \leftrightarrow \tilde{\gamma}_{k} \tilde{P}, I \leftrightarrow \tilde{I}, P \leftrightarrow \tilde{P}
$$

where $k, l=0,1,2,3,4$,

$$
\tilde{\gamma}_{k}=\left(\begin{array}{cc}
\gamma_{k} & 0 \\
0 & \gamma_{k}
\end{array}\right), \quad \tilde{P}=\left(\begin{array}{cc}
I & 0 \\
0 & -I
\end{array}\right), \quad \tilde{I}=\left(\begin{array}{cc}
I & 0 \\
0 & I
\end{array}\right)
$$

$\gamma_{k}$ are the real Dirac matrices

$$
\begin{gathered}
\gamma_{0}=\left(\begin{array}{cc}
0 & \sigma_{1} \\
-\sigma_{1} & 0
\end{array}\right), \quad \gamma_{1}=\left(\begin{array}{ll}
0 & I \\
I & 0
\end{array}\right), \quad \gamma_{2}=\left(\begin{array}{cc}
0 & -i \sigma_{2} \\
i \sigma_{2} & 0
\end{array}\right), \\
\gamma_{3}=\left(\begin{array}{cc}
I & 0 \\
0 & -I
\end{array}\right), \quad \gamma_{4}=\left(\begin{array}{cc}
0 & \sigma_{3} \\
-\sigma_{3} & 0
\end{array}\right),
\end{gathered}
$$

0 and $I$ are zero and unit matrices of appropriate dimension.

Algebra $g l(4, C)$ includes 32 real matrices $\left\{\tilde{\gamma}_{k}, \tilde{\gamma}_{k} \tilde{\gamma}_{l}, \tilde{P} \tilde{\gamma}_{k}, \tilde{P} \tilde{\gamma}_{k} \tilde{\gamma}_{l}, \tilde{P}, \tilde{I}\right\}$, moreover, 20 of them are symmetric and 12 antisymmetric.

Antisymmetric matrices are no diagonalizable over the field of real numbers, thus it is sufficient to consider only symmetric ones, i.e.,

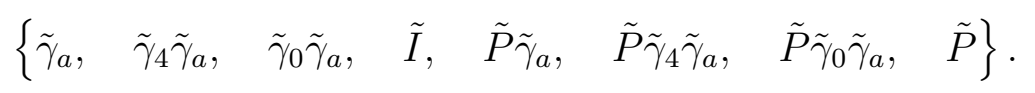

The related set of symmetries of equation (1) is

$$
\hat{S}=\left\{S_{5 a}, S_{4 a}, S_{0 a}, \hat{I}, \hat{S}_{5 a}, \hat{S}_{4 a}, \hat{S}_{0 a}, P\right\}
$$

The trivial unit symmetry operator cannot be used to reduce equation (1), thus without loss of generality we can exclude from (33) the unit matrix. We exclude also all terms which include $\gamma_{4}$ inasmuch as

$$
\tilde{\gamma}_{4} \tilde{\gamma}_{a}=U \tilde{\gamma}_{0} \tilde{\gamma}_{a} U^{\dagger}, \quad \tilde{P} \tilde{\gamma}_{4} \tilde{\gamma}_{a}=U \tilde{P} \tilde{\gamma}_{0} \tilde{\gamma}_{a} U^{\dagger}
$$

where $U=\frac{1}{\sqrt{2}}\left(1+\tilde{\gamma}_{4} \tilde{\gamma}_{0}\right)$. The corresponding symmetries of equation (1) are connected by the equivalence transformation (31).

Thus, starting with the algebra $g l(4, C)$ we select the diagonal matrix $\tilde{P}$ and 12 symmetric matrices

$$
\tilde{\gamma}_{a}, \quad \tilde{\gamma}_{0} \tilde{\gamma}_{a}, \quad \tilde{P} \tilde{\gamma}_{a}, \quad \tilde{P} \tilde{\gamma}_{0} \tilde{\gamma}_{a},
$$

whose number cannot be reduced using transformation (35). In accordance with (34), the corresponding symmetries of the SP equation are $P$ and

$$
S_{5 a}, \quad S_{0 a}, \quad \hat{S}_{5 a}, \quad \hat{S}_{0 a} .
$$


Any of these 13 symmetries are diagonalizable and generate reductions of the SP equation to two uncoupled subsystems. An example of such a reduction is given in Section 4.

We note that the sets (34), (37) can be obtained by the following reductions of algebra $g l(4, C)$ :

$$
g l(4, C) \rightarrow s p(2, C) \rightarrow s o(1,3) \oplus s o(1,3)
$$

A basis of the subalgebra $s p(2, C)$ is generated by the set $S=\left\{\tilde{\gamma}_{\mu} \tilde{\gamma}_{\nu}, \tilde{\gamma}_{4} \tilde{\gamma}_{\mu}, \tilde{P} \tilde{\gamma}_{\mu} \tilde{\gamma}_{\nu}, \tilde{P}_{\gamma_{4}} \tilde{\gamma}_{\mu},\right\}$ which satisfy the condition $\left(Q \tilde{\gamma}_{0} \tilde{\gamma}_{4}\right)^{\dagger}=Q \tilde{\gamma}_{0} \tilde{\gamma}_{4}, Q \in S$. In other words, multiplying any element of $S$ by $\tilde{\gamma}_{0} \tilde{\gamma}_{4}$ we obtain the set of symmetric matrices (33). Matrices $\frac{1}{4}(1 \pm \tilde{P}) \tilde{\gamma}_{\mu} \tilde{\gamma}_{\nu}$ and $\tilde{\gamma}_{0} \tilde{\gamma}_{4}$ form commuting subalgebras so $(1,3)$ and one-dimensional algebra which we denote by $d(1)$. Products of matrices belonging to $S$ with $\tilde{\gamma}_{0} \tilde{\gamma}_{4}$ generate the set of 12 symmetric matrices (36).

Let us present the explicit form of operators which reduce the SP equation:

\begin{tabular}{l|l|l|l}
$\mathrm{N}$ & Symmetry & Parities of $A_{\mu}$ & Reducing operator \\
\hline 1. & $S_{5 \alpha}=\sigma_{2} \theta_{2}$ & $A_{2}\left(\theta_{\alpha} x\right)=-A_{2}(x), \alpha \neq 2$ & \\
& & $A_{k}\left(\theta_{\alpha} x\right)=A_{k}(x), k \neq \alpha$ & $U=\frac{1}{\sqrt{2}}\left(1+\sigma_{3} S_{5 a}\right)$ \\
2. & $S_{53}=\sigma_{3} \theta_{3}$ & $A_{3}\left(\theta_{3} x\right)=-A_{3}(x)$ & \\
& & $A_{k}\left(\theta_{3} x\right)=A_{k}(x), k \neq 3$ & $U=\frac{1}{2}\left(1+\sigma_{2}\right)\left(1+\sigma_{1} S_{53}\right)$ \\
3. & $S_{0 \alpha}=i \sigma_{2} i \sigma_{\alpha} c \theta_{0} \theta_{\alpha}$, & $A_{\sigma}\left(\theta_{0} \theta_{\alpha} x\right)=A_{\sigma}(x), \sigma=0, \alpha$, & $U=\frac{1}{\sqrt{2}}\left(1+\sigma_{3} S_{0 a}\right)$ \\
& $\alpha \neq 2$ & $A_{\nu}\left(\theta_{0} \theta_{\alpha} x\right)=-A_{\nu}(x), \nu \neq 0, \alpha$ & \\
4. & $S_{02}=i c \theta_{0} \theta_{2}$, & $A_{\sigma}\left(\theta_{0} \theta_{\alpha} x\right)=A_{\sigma}(x), \sigma=0,2$, & $U=\frac{1}{\sqrt{2}}\left(1+i \theta_{0} \theta_{2}\right)$ \\
& & $A_{\nu}\left(\theta_{0} \theta_{\alpha} x\right)=-A_{\nu}(x), \nu \neq 0,2$ & \\
5. & $\hat{S}_{5 a}=\sigma_{a} \theta_{b c}$ & $A_{k}\left(\theta_{b c} x\right)=A_{k}(x), k \neq b, c$, & $U=\frac{1}{\sqrt{2}}\left(1+\hat{S}_{5 a}\right)$ \\
& $a \neq 3, b, c$ & $A_{k}\left(\theta_{b c} x\right)=-A_{k}(x), k=b, c$ & \\
6. & $\hat{S}_{53}=\sigma_{3} \theta_{12}$ & $A_{k}\left(\theta_{12} x\right)=A_{k}(x), k \neq 1,2$, & $U=\frac{1}{2}\left(1+i \sigma_{2}\right)\left(1+\sigma_{1} \hat{S}_{53}\right)$ \\
& & $A_{k}\left(\theta_{12} x\right)=-A_{k}(x), k=1,2$ & \\
7. & $\hat{S}_{0 a}=i \sigma_{2} \theta \theta_{a}$ & $A_{b}\left(\theta \theta_{a}\right)=-A_{b}(x), b \neq a$, & $U=\frac{1}{\sqrt{2}}\left(1+\sigma_{3} \hat{S}_{0 a}\right)$ \\
& $a \neq 2$ & $A_{a}\left(\theta \theta_{a}\right)=A_{a}(x)$ & \\
8. & $\hat{S}_{02}=i \sigma_{2} \theta \theta_{2}$ & $A_{b}\left(\theta \theta_{2}\right)=-A_{b}(x), b \neq 2$ & $U=\frac{1}{\sqrt{2}}\left(1+i \theta_{1} \theta_{3}\right)$ \\
& & $A_{2}\left(\theta \theta_{2}\right)=A_{2}(x)$ & \\
9. & $\hat{P}=\theta_{1} \theta_{2} \theta_{3}$ & $A_{0}\left(\theta \theta_{0} x\right)=A_{0}(x)$, & $U=I$ \\
& $A_{a}\left(\theta \theta_{0} x\right)=-A_{a}(x), a \neq 0$ &
\end{tabular}

The operators $U$ presented in the table diagonalize the corresponding symmetries and reduce equation (1) to two uncoupled subsystems. Such a reduction can be made in analogy with (25)-(27).

\section{Multiple reductions}

If equation (1) admits two or more commuting symmetries from set (34), we can diagonalize them simultaneously. Such a diagonalization generates a product of reductions 
of equation (1), i.e., multiple reductions. An example of a multiple reduction is given by relations (29), (30).

To enumerate all multiple reductions generated by discrete symmetries it is sufficient to find all nonequivalent subsets of commuting operators from set (34). Taking into account that operators (34) with non-coinciding indices commute we find the following doublets and triplets of commuting symmetries

$$
\begin{gathered}
\left\{S_{5 a}, S_{0 b}\right\}, \quad\left\{\hat{S}_{5 a}, \hat{S}_{0 b}\right\}, \quad\left\{\hat{S}_{5 a}, S_{0 b}\right\}, \\
\left\{S_{5 a}, \hat{P}\right\}, \quad\left\{S_{0 a}, \hat{P}\right\}, \\
\left\{S_{5 a}, S_{0 b}, P\right\}
\end{gathered}
$$

where $a$ and $b$ are fixed, $a \neq b$. Using the equivalence relations $\left\{Q_{A}, Q_{B}\right\} \sim$ $\left\{Q_{A}, Q_{A} Q_{B}\right\} \sim\left\{Q_{B}, Q_{A} Q_{B}\right\}, \quad Q_{a}, Q_{B} \in \hat{S}$ and relations (38), it is easily to prove that all other doublets and triplets of commuting symmetries (34) are equivalent to ones enumerated in (39).

Equation (1) admits a doublet of symmetries from set (39) provided the vectorpotential $A_{\mu}(x)$ satisfies two (or three) the corresponding parity conditions present in the table. If so, the related equation (1) can be decoupled to four (or eight) uncoupled subsystems. The corresponding transformation operators is a product of operators $U$ given in the table. In accordance with (39) there exist 24 different reductions to four subsystems and 6 different reductions to eight subsystems.

We notice that the sets (39a) can be obtained by the following continuation of algebraic reductions (36):

$$
s o(1,3) \oplus s o(1,3) \rightarrow s o(3)
$$

where subalgebras $s o(3)$ have to contain at least one antiunitary (i.e., including complex conjugation) operator. These subalgebras are generated by products of operators (39a) with $S_{40} P$.

The sets (39b,c) include the parity operator which does not appear in reductions (40).

\section{Symmetries and reductions of the Schrödinger equation}

Here we consider the usual Schrödinger equation for a spinless particle

$$
\hat{L} \psi \equiv\left[p_{0}-\left(\frac{1}{2 m} \pi^{2}+e A_{0}\right)\right] \psi=0
$$

and describe its possible reductions with using discrete symmetries.

Let the vector-potential $A(x)$ satisfies one of relations (3a), (10a), (10c), (10e). The corresponding equation (41) admits one of the following symmetry operators

$$
Q_{0}=c \theta_{0}, \quad Q_{0 a}=c \theta_{0} \theta_{a}, \quad Q_{40}=c \theta_{0} \theta_{1} \theta_{2} \theta_{3}, \quad Q_{4 a}=c \theta_{0} \theta_{b} \theta_{c}
$$

respectively. Here $c$ and $\theta_{\mu}$ are operators defined in (5), $b, c \neq a$. 
Using the transformation $Q_{A} \rightarrow U_{A} Q_{A} U_{A}^{\dagger}=c$ where $U_{A}=\frac{1}{2}(1-i)\left(1+i c Q_{A}\right)$ (the multiindex A takes the values $A=0,0 a, 40,4 a$ ), we can reduce any of symmetries (42) to the operator of complex conjugation. It means that the related transformed equation (41) $\hat{L}_{A}^{\prime} \psi_{A}^{\prime}=0$ (where $\hat{L}_{a}^{\prime}=U_{A} L U_{A}^{\dagger}, \quad \psi_{A}^{\prime}=U_{A} \psi$ ) decouples to two independent subsystems for real and imaginary parts of $\psi_{A}^{\prime}$.

If $A(x)$ satisfies one of relations (3b), (10b), (10c) then equation (41) admits one of the symmetries

$$
Q_{A}=\theta_{a}, \quad Q_{a b}=\theta_{a} \theta_{b}, \quad Q_{123}=\theta_{1} \theta_{2} \theta_{3}
$$

respectively and can be reduced to uncoupled subsystems for $\psi_{ \pm}=\frac{1}{2}\left(1 \pm Q_{A}\right)$ where multiindex $A$ takes the values $a, a b, 123$.

All operators (42), (43) commute, so we can make multiple reductions provided $A(x)$ satisfies two or more of relations (3), (10) simultaneously.

\section{Extended and generalized SUSY}

All discrete symmetries analyzed in Sections 3 are evidently valid for the particular case $A_{0}=0, g=2$. The related $\mathrm{SP}$ equation

$$
i \frac{\partial}{\partial t} \psi=\hat{H} \psi, \quad \hat{H}=\frac{1}{2 m}\left(\pi^{2}-e \vec{\sigma} \cdot H\right)
$$

admits additional symmetry operators which are called supercharges.

For any $A_{a}(\vec{x})$, there exists the following supercharge for equation (44) (whose square is equal to $\hat{H}): Q_{1}=\frac{1}{\sqrt{2 m}}(\vec{\sigma} \cdot \vec{\pi})$.

If vector-potential $\vec{A}(\vec{x})$ has definite parities, i.e., satisfies relations (13c) or (14c), than there exist the second supercharge $Q_{2}=i P Q_{1}$ or $Q_{2}=i \hat{P} Q_{1}$ respectively [5]. These supercharges satisfy the superalgebra [9]

$$
\begin{gathered}
\{Q, \bar{Q}\}=2 \hat{H}, \quad Q^{2}=(\bar{Q})^{2}=0 \\
{[Q, \hat{H}]=0,[\bar{Q}, \hat{H}]=0}
\end{gathered}
$$

where $Q=\frac{1}{\sqrt{2}}\left(Q_{1}+i Q_{2}\right), \quad \bar{Q}=\frac{1}{\sqrt{2}}\left(Q_{1}-i Q_{2}\right)$.

It was demonstrated in $[2,6,7]$ that superalgebra (45) can be extended provided $\vec{A}(\vec{x})$ satisfies two or more relations (13), (14) simultaneously. Using algebras of discrete symmetries discussed in Section 3, it is easy to construct classes of models with extended (i.e., including more than two supercharges) SUSY, which are wider than classes considered in papers $[2,7]$, where only parities of type (13) (but no of type (14)) where analyzed. Here we present some examples.

Let $\vec{A}(\vec{x})$ satisfies relations (19). Then equation (44) admits four supercharges

$$
\begin{array}{cl}
Q^{1}=\frac{1}{2 \sqrt{m}}\left(1+C \Gamma_{b}\right) \vec{\sigma} \cdot \vec{\pi}, & \bar{Q}^{1}=\frac{1}{2 \sqrt{m}}\left(1-C \Gamma_{b}\right) \vec{\sigma} \cdot \vec{\pi}, \\
\bar{Q}^{2}=\frac{1}{2 \sqrt{m}}\left(i R_{a}+C \Gamma_{c}\right) \vec{\sigma} \cdot \vec{\pi}, & \bar{Q}^{2}=\frac{1}{2 \sqrt{m}}\left(i R_{a}-C \Gamma_{c}\right) \vec{\sigma} \cdot \vec{\pi}
\end{array}
$$

Supercharges (46) with non-coinciding indices anticommute, the other commutation and anticommutation relations for them are analogous to (45). 
Thus, relations (19) define a class of vector-potentials for which equation (44) admits extended $N=4$ SUSY. In addition to (22), this class includes such important particular cases as the constant magnetic, solenoidal, and magnetic dipole field. The related vectorpotentials have the forms

$$
\begin{gathered}
A_{a}=0, \quad A_{b}=e H x_{c} / 2, \quad A_{c}=-e H x_{b} / 2, \\
A_{a}=0, \quad A_{b}=-\lambda_{2} \frac{x_{c}}{x_{b}^{2}+x_{c}^{2}}, \quad A_{c}=\lambda_{2} \frac{x_{b}}{x_{b}^{2}+x_{c}^{2}}
\end{gathered}
$$

and

$$
A_{a}=0, \quad A_{b}=-\lambda_{3} \frac{x_{c}}{\left(\sqrt{x_{b}^{2}+x_{c}^{2}}\right)^{3}}, \quad A_{c}=\lambda_{3} \frac{x_{b}}{\left(\sqrt{x_{b}^{2}+x_{c}^{2}}\right)^{3}}
$$

respectively (we do not specify coupling constants $\lambda_{a}$ ). These potentials obviously satisfy relations (19).

The other example of the vector-potential which corresponds to magnetic octopole field (21) and generate extended SUSY for equation (44) was presented in paper [7]. The related parities of $\vec{A}(\vec{x})$ are defined by relations (18).

\section{Discussion}

We investigate algebraic structures generated by discrete symmetries of the SP equation and prove that they form a basis of algebra $g l(4, C)$.

These internal symmetries can be used to predict the related degeneration of Hamiltonian spectra and to reduce the SP equation to uncoupled subsystems. To enumerate all possibilities arising in this way make reductions of algebra $g l(4, C)$ on its subalgebras, refer to Sections 5,6.

The other interesting application of discrete symmetries is connected with searching for extended or generalized [4] supersymmetry of the SP equation. The possibility of using of different kinds of parity operators to construct supercharges was discovered long time ago [5]. Recently this idea was applied in searching for extended SUSY of the Dirac $[2,6]$ and SP [7] equations.

The symmetry algebra $g l(4, C)$ opens additional possibilities in searching for extended SUSY. Indeed, basis elements of this algebra are involutions which either commute or anticommute with the "standard" supercharge $\sigma \cdot \vec{\pi}$, so their products with $\sigma \cdot \vec{\pi}$ can generate new supercharges. The above mentioned involutions can be applied also to construct models admitting generalized SUSY [4].

We notice once more, that the symmetry algebra $g l(4, C)$ includes reflections and pure rotations as well. It is possible to generate models with extended SUSY asking for definite properties of $\vec{A}(\vec{x})$ w.r.t. these rotations. Such models cannot be obtained either in approach [5] or [7].

For example, let the vector-potential satisfies relations (13b) for all possible values of indices $a, b$, but does not satisfy either relations (12a), (12c) or (13). Then equation (44) admits three (hermitian) supercharges $Q_{1}=i P_{23} Q, Q_{2}=i P_{31} Q, Q_{3}=i P_{12} Q$, where $Q=\frac{1}{\sqrt{2}} \vec{\sigma} \cdot \vec{\pi}$ and $P_{a b}$ are operators defined by relations (13b). An example of the external field with such symmetries is given by the superposition of potentials (21) and (24). 
The symmetry algebra $g l(4, C)$ is admitted by the SP equation for a wide class of external fields, whose potentials satisfy relations (3). This class includes the Coulomb potential and different kinds of vector-potentials whose space components are odd functions of $t$ and $x_{a}$. An example of such a potential is given in Section 2 .

Algebra of discrete symmetries of the Dirac equation (which is equivalent to $g l(8, R)$ ) was analyzed in papers $[2,3]$.

I am indebted to the anonimous referee for critical comments and helpful suggestions.

This paper is supported by the Ukrainian DFFD foundation through grant No. $1.4 / 356$.

\section{References}

[1] P. Olver, Application of Lie Groups to Differential Equations (N.Y., Springer, 1986); N.Ch. Ibragimov, Transformation Groups in Mathematical Physics (Nauka, Moscow, 1983).

[2] J. Niederle and A.G. Nikitin, J. Phys. A 30, 999 (1997).

[3] J. Niederle and A.G. Nikitin, J. Nonlin. Math. Phys. 4, 436 (1997).

[4] N. B. Borisov, K. N. Illinski, and V. M. Uzdin, Phys. Lett. A 169, 422 (1992); Teor. Mat. Fiz. 94, 418 (1993);

A. D. Dolgallo and K. N. Illinski, Ann. Phys. 236, 219 (1994).

[5] L. E. Gendenshtein, Yad. Fiz. 41, 261 (1985);

L. E. Gendenshtein and N. V. Krive, Usp. Fiz. Nauk 146, 583 (1985).

[6] A.G. Nikitin, On Extended Supersymmetries and Parasupersymmetries, In: X International Conference "Problems of Quantum Field Theory", JINR E2-96-369, Dubna, 1996 ;

A.G.Nikitin, On Extended Supersymmetries in Quantum Mechanics, In: GROUP21 Physical applications and mathematical aspects of geometry, groups and algebras, Vol. 1, Eds. H.-D. Doebner, W. Sherer, P. Natterman, World Scientific, Singapoore, 1997, p. 509-514.

[7] V.M. Tkachuk, S.I. Vakarchuk, Phys.Lett. A 228, 141 (1997).

[8] E.P. Wigner, Unitary Representations of Lorentz Group Including Reflections, in: Theoretical Concepts and Methods in Elementary Particle Physics, Lect.Istanbul School of Theor.Phys. (Gordon and Breach, 1964).

[9] E. Witten, Nucl. Phys. B 185, 563 (1981). 